\title{
THE GEANT4-BASED ATLAS FAST ELECTROMAGNETIC SHOWER SIMULATION
}

\author{
E BARBERIO $^{1}, \mathrm{~J}_{\text {BOUDREAU }}^{2}$, B BUTLER $^{3}, \mathrm{~S} \mathrm{~L} \mathrm{CHEUNG}^{4}, \mathrm{~A}^{5}$ DELL'ACQUA $^{5}$, \\ A DI SIMONE ${ }^{5}$, W EHRENFELD ${ }^{6,7}$, M V GALLAS $^{5}$, A GLASOW $^{7}$, E HUGHES $^{8}, Z$ \\ MARSHALL $^{8,9}$, J MUELLER $^{2}$, R PLACAKYTE $^{7}$, A RIMOLDI $^{10}$, P SAVARD $^{4}$, \\ V TSULAIA $^{2}$, A WAUGH $^{11}, \mathrm{C} \mathrm{C} \mathrm{YOUNG}^{3}$ \\ ${ }^{I}$ School of Physics, University of Melbourne, Parkville, Victoria, 3010, Australia \\ ${ }^{2}$ Department of Physics and Astronomy, University of Pittsburgh, 3941 O'Hara Street, \\ Pittsburgh, PA 15260, USA \\ ${ }^{3}$ Stanford Linear Accelerator Center, 2575 Sand Hill Road, Menlo Park, CA 94025, USA \\ ${ }^{4}$ Department of Physics, University of Toronto, 60 Saint George Street, Toronto, M5S \\ 1A7, Ontario, Canada \\ ${ }^{5}$ European Laboratory for Particle Physics, CERN, 1211 Geneva 23, Switzerland \\ ${ }^{6}$ Institut für Experimentalphysik, Universität Hamburg, Luruper Chaussee 149, 22761 \\ Hamburg, Germany \\ ${ }^{7}$ Deutsches Elektronen-Synchroton, DESY, Notkestrasse 95, 22603 Hamburg, Germany \\ ${ }^{8}$ Nevis Laboratory, Columbia University, 136 So. Broadway, Irvington, NY 10533, USA \\ ${ }^{9}$ Physics Department, California Institute of Technology, Pasadena, CA 91125, USA \\ ${ }^{10}$ Dipartimento di Fisica Nucleare e Teorica, Universita di Pavia and I.N.F.N., Via A \\ Bassi 6, 27100 Pavia, Italy \\ ${ }^{11}$ Falkiner High Energy Physics Group, School of Physics, University of Sydney, Sydney,
} NSW 2006, Australia

We present a three-pronged approach to fast electromagnetic shower simulation in ATLAS. Parameterisation is used for high-energy, shower libraries for medium-energy, and an averaged energy deposition for very low-energy particles. We present a comparison between the fast simulation and full simulation in an ATLAS Monte Carlo production.

\section{Introduction}

ATLAS [1], one of two general-purpose experiments designed to collect data from the Large Hadron Collider, has implemented a detector simulation within the Athena software framework [2] based on GEANT4 [3]. However, owing to the complexity of both GEANT4 itself and the ATLAS detector geometry, the standard simulation ("full simulation") can take upwards of twenty minutes to simulate single physics events. The fast simulation package speeds up the simulation of electromagnetic (EM) showers within the calorimeters. 


\section{Motivation}

The CPU time spent for simulating the ATLAS subdetectors motivated the development of a fast shower simulation. Table 1 shows the CPU time spent on simulation of various particles as they passed through the subdetectors for QCD dijet events. The vast majority of time is spent on EM particles in the calorimeters. No other detector element contributes more than $6 \%$ of the total CPU time.

Energy spectra of particles entering the sampling portion of the calorimeters in the same dijet events were used to determine the most important energies to study in the fast simulation. Several orders of magnitude more particles enter the calorimeter with energies of a few tens of $\mathrm{MeV}$ than at energies over 500 $\mathrm{MeV}$; the simulation of low-energy particles drives the total simulation time. An order of magnitude more photons below $10 \mathrm{MeV}$ appear in typical events than all other particles combined. At high energies, photons pair produce after only a few steps, so that treating the subsequent electron-positron pair suffices to speed up the simulation. Below about $10 \mathrm{MeV}$, this is no longer the case.

Table 1. CPU time per event for full and fast simulation by subdetector and particle type for simulation of a QCD dijet sample with a $280-560 \mathrm{GeV}$ leading jet. Systematic and statistical uncertainties are each at the few percent level. Timer overhead, $\sim 5 \%$, was removed. "Other Systems" mostly comprises beam pipe, detector services, and shielding. Neutrons dominate "Other Particles" ( $90 \%)$.

\begin{tabular}{ccc}
\hline Detector Subsystem & Full Sim. Time [kSI2Ks] & Fast Sim. Time [kSI2Ks] \\
\hline Tracker & 44 & 38 \\
EM Barrel Calorimeter & 91 & 23 \\
EM Endcap Calorimeter & 393 & 107 \\
Forward Calorimeter & 155 & 55 \\
Hadronic Barrel Calorimeter & 29 & 27 \\
Hadronic Endcap Calorimeter & 50 & 47 \\
Muon System & 21 & 21 \\
Other Systems & 124 & 89 \\
\hline Total Event & $\mathbf{9 0 7}$ & $\mathbf{4 0 6}$ \\
\hline Particle Type & Full Sim. Time [kSI2Ks] & Fast Sim. Time [kSI2Ks] \\
\hline Electrons and Positrons & 344 & 124 \\
Photons & 259 & 88 \\
Other Particles & 304 & 194 \\
\hline Total Event & $\mathbf{9 0 7}$ & $\mathbf{4 0 6}$ \\
\hline
\end{tabular}




\section{Fast Simulation}

The fast shower framework comprises three methods: a high-energy $(>10 \mathrm{GeV})$ parameterisation based on [4]; a medium-energy $(<1 \mathrm{GeV})$ shower library approach, consisting of pre-simulated particle showers; and a "killing" approach that recreates the average energy and position of the one hit typically created by sufficiently low-energy $(<10 \mathrm{MeV})$ particles.

As a particle moves through the detector, each is separately examined by the fast simulation package. When a particle enters the homogeneous sampling region of a calorimeter, its type is checked. Only photons, electrons, and positrons can trigger the fast simulation. If the shower's energy is more than $95 \%$ contained, longitudinally and transversely, in that region, and the initial particle's energy is in the chosen range, the particle is accepted. If any test fails, GEANT4 moves that particle one more step, and the particle is retested.

If the particle satisfies all criteria, one of the three fast shower methods is applied. The original direction of the particle determines the core of the eventual shower; systematic effects from the magnetic field are taken to be small.

Each method was studied individually to assess its impact on the simulation time and accuracy in reproducing the full simulation. Because of the energy spectrum of particles entering the calorimeters, the shower libraries and "killing" are applied more frequently than the parameterisation.

\section{Shower Libraries Method}

Particles captured by the fast simulation in the appropriate energy range are replaced by a shower from a pre-simulated library, rotated and scaled to match the primary particle [4]. Shower libraries are generated in bins of pseudorapidity and energy for electrons and photons. Only hits in the sensitive detectors are stored. The binning reproduces the fine structure in the calorimeters.

\section{5. "Killing" Method}

Low-energy $(<10 \mathrm{MeV})$ electrons deposit on average only one hit in the sensitive detectors. The properties of that hit are all that must be reproduced. A step is taken in the direction of the original particle, with a varied length. The particle's energy is scaled to recreate the energy response of the calorimeter. Absorber and active material are not separated, providing a geometric energy fluctuation. No containment test is applied to avoid a bias near detector cracks. 


\section{Parameterisation Method}

For electrons and positrons above a sufficiently high energy, around $10 \mathrm{GeV}$, the detector appears sufficiently homogeneous to apply a shower parameterisation. The parameterisation takes small steps in the direction of the original particle, depositing energy according to several tuned functions as it goes.

The longitudinal profiles of showers were parameterised following the approach in [5] and normalized with an energy scale for each subdetector. The radial profile is a 2-dimensional function of radius and depth in the shower, as in $[5,6]$, normalized by the longitudinal profile. Energy is deposited in GEANT4 hits [3] in order to mimic full simulation. Fluctuations are introduced in three separate places, representing the random characteristics of shower length and shape, the sampling resolution of the calorimeter, and the geometric fluctuations in the energy collected.

\section{Full and Fast Simulation Comparison}

The fast simulation has been tuned to and compared with full simulation Monte Carlo in Athena release 13.0.30. Simulation time for dijet events is reduced by a factor of two to three, as seen in Table 1.

Average energy deposition in the sensitive detector is well reproduced by the fast simulation. Figure 1 displays fast and full simulation energy depositions in the forward calorimeter for primary electrons of several initial energies and several initial pseudorapidities. The ratio of the energy deposited in the first sampling of the EM calorimeter to that in the second provides an important

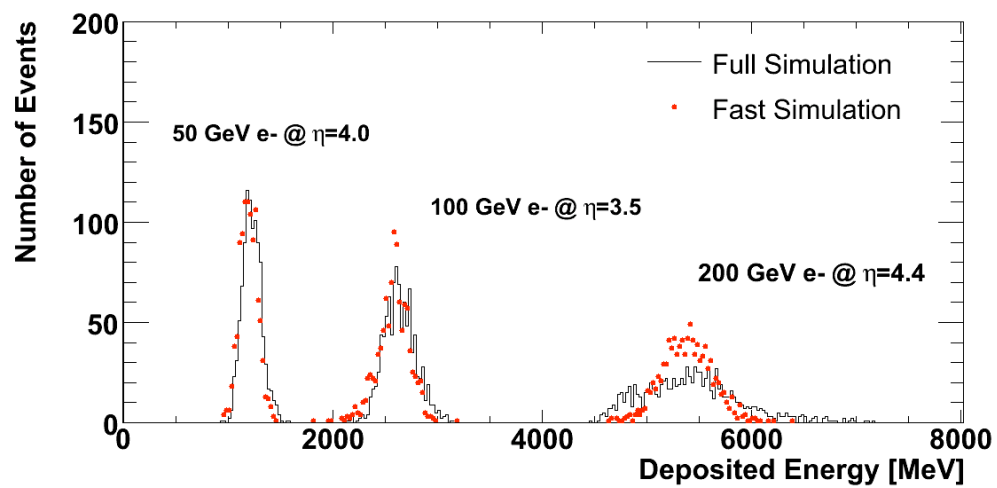

Figure 1: Energy deposition in the ATLAS forward EM calorimeter for primary electrons of various energies. Full simulation is presented in black (solid) and fast simulation in red (dots). 


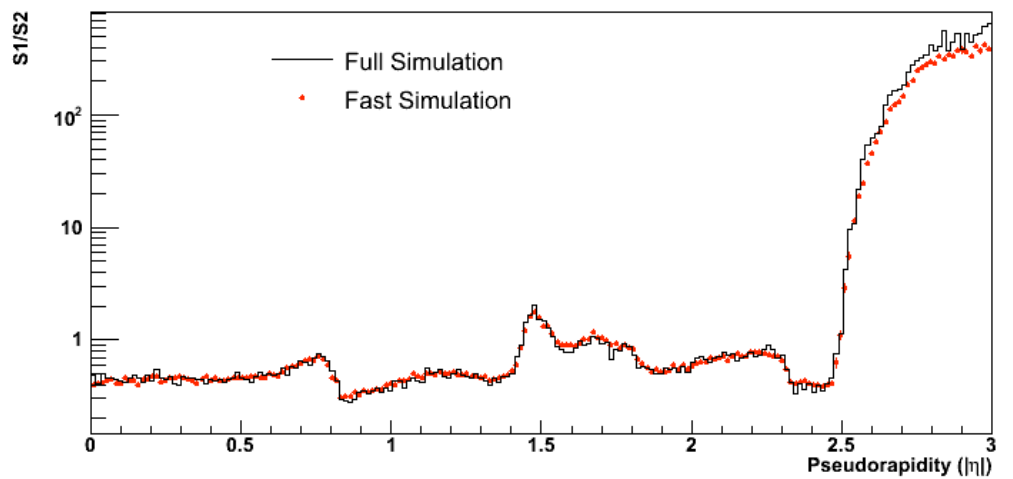

Figure 2: The ratio of energy deposition in the first sampling layer of the ATLAS EM calorimetry to that in the second layer for $50 \mathrm{GeV}$ primary electrons, as a function of pseudorapidity, is presented for full (red pluses) and fast (blue stars) simulation. The EM barrel calorimeter ends at $|\eta|=1.4$.

check for electron and photon identification. The quantity is reproduced at the few percent level, except at high pseudorapidity (Fig. 2).

\section{Conclusions}

We have presented a fast simulation approach for the ATLAS EM calorimetry, comprising parameterisation of high-energy particles, shower libraries for medium-energy particles, and "killing" for low-energy particles. A comparison with full simulation of physics observables and CPU time is included.

Work is ongoing to improve physics agreement and further reduce simulation time. The extension of several of these models to additional subdetectors and particles is currently being tested.

\section{References}

1. ATLAS Collaboration, The ATLAS Experiment at the CERN Large Hadron Collider, 2007.

2. ATLAS Collaboration, Athena - The ATLAS Common Framework, Version 8, http://atlas-computing.web.cern.ch/atlascomputing/documentation/ swDoc/AthenaDeveloperGuide-8.0.0-draft.pdf, CERN, 2006.

3. J. Allison et al., IEEE Transactions on Nuclear Science 53270 (2006).

4. E. Barberio, et al. "Fast shower simulation in ATLAS Calorimeter." Proceedings of the CHEP 2007 Conference in Vancouver, Canada, Aug 2007.

5. G. Grindhammer, M Rudowicz, S. Peters, NIM A290, 469 (1990); G. Grindhammer, S. Peters, hep-ex/0001020; Fast Simulation Working Group, https://twiki.cern.ch/twiki/bin/view/Atlas/AtlasShowerParam.

6. J. del Peso, E. Ros, NIM A306, 485 (1991). 